\title{
Using Genetic Algorithms to Find Person-Specific Gabor Feature Detectors for Face Indexing and Recognition
}

\author{
Sreekar Krishna, John Black, and Sethuraman Panchanathan \\ Center for Cognitive Ubiquitous Computing (CUbiC), \\ Arizona State University, Tempe AZ- 85281 \\ Tel: 480326 6334, Fax Number: 4809651885 \\ Sreekar.Krishna@asu.edu
}

\begin{abstract}
In this paper, we propose a novel methodology for face recognition, using person-specific Gabor wavelet representations of the human face. For each person in a face database a genetic algorithm selects a set of Gabor features (each feature consisting of a particular Gabor wavelet and a corresponding $(x, y)$ face location) that extract facial features that are unique to that person. This set of Gabor features can then be applied to any normalized face image, to determine the presence or absence of those characteristic facial features. Because a unique set of Gabor features is used for each person in the database, this method effectively employs multiple feature spaces to recognize faces, unlike other face recognition algorithms in which all of the face images are mapped into a single feature space. Face recognition is then accomplished by a sequence of face verification steps, in which the query face image is mapped into the feature space of each person in the database, and compared to the cluster of points in that space that represents that person. The space in which the query face image most closely matches the cluster is used to identify the query face image. To evaluate the performance of this method, it is compared to the most widely used subspace method for face recognition: Principle Component Analysis (PCA). For the set of 30 people used in this experiment, the face recognition rate of the proposed method is shown to be substantially higher than PCA.
\end{abstract}

\section{Introduction}

Faces are an important biometric, and many computer algorithms have been proposed to identify face images. However, existing face recognition algorithms are not very robust with respect to pose angle or illumination angle variations. Humans are much better at recognizing faces when faced with these types of variations. This has prompted researchers to more closely study the ways in which humans recognize faces, and face recognition has become a proving ground for artificial intelligence researchers who are attempting to simulate human pattern recognition with computer algorithms.

Face recognition algorithms can be broadly classified into holistic methods and feature-based methods. Holistic methods attempt to recognize a face without 
subdividing it into component parts, while feature-based methods subdivide the face into components (i.e. features) and analyze each feature, as well as its spatial location with respect to other features. The performance of holistic face recognition algorithms has been shown to be highly variable with respect to variations in pose angle, illumination angle, and facial expressions. Failures to achieve more robust face recognition using the holistic methods have motivated many researchers to study feature-based methods. This paper describes our own attempt to develop a featurebased method of face recognition that provides a higher level of performance than that of the existing holistic methods.

The rest of the paper is organized as follows: Section 2 discusses past research in the use of Gabor filters and Genetic Algorithms (GAs) in face recognition. Section 3 discusses the theoretical basis for our research. Section 4 describes the methodology we have used, including the implementation details of (1) the Gabor wavelets that we used to extract facial features, (2) the genetic algorithm that we used to select the Gabor feature detectors, and (3) the experiments that we used to evaluate the performance of the proposed algorithm. Section 5 presents the results of our experiments, and Section 6 discusses those results. Section 7 concludes the paper, and includes a discussion of future work.

\section{Related Work}

Classical methods of face recognition have employed statistical analysis techniques such as the Principle Component Analysis (PCA) [2] and Linear Discriminant Analysis (LDA) [3] which are logical extensions of the data analysis methods developed to investigate large datasets. These methods treat each face image as a point in a high-dimensional space, and try to associate multiple views of a person's face with a distinct cluster in that space. The problem with using these statistical methods is that small variations in capture conditions tend to scatter face images of each person across a wide expanse of this space, making it difficult to discern a distinct cluster for each person.

Faced with this problem, many researchers have attempted to extract localized facial features. Among the many available feature extractors, Gabor wavelets have been popular - possibly due to the fact that Gabor wavelets model the receptive fields of the simple cells [4]. Shen et al. [5] used Gabor filters in combination with a Kernel Direct Discriminant Analysis (KDDA) subspace as a classifier, and Liu et al proposed using Gabor filters in an Enhanced Fisher Linear Discriminant Model [7] and with Independent Component Analysis (ICA) [6]. However, none of these methods specifically select feature detectors (or the locations of their application) based on the salient features of faces. There exists some face recognition research that does take into account the localities of salient facial features [8] [9]. However, these methods rely on a human to select facial feature locations manually, leaving open the question of how much this human contribution influences the results.

Genetic Algorithms, (GAs) have been used in face recognition to search for optimal sets of features from a pool of potentially useful features that have been extracted from the face images. Liu et al. [10] used a GA to search for optimal 
components from a pool of independent components, while Xu et al. [11] used a GA to search for the optimal components in a pool of Kernel Principle Components.

In each of the cases described above, all of the faces in a database were indexed with a single feature set. We believe that this approach imposes a fundamental and unnecessary constraint on the recognition of faces. We suspect that people first learn to recognize faces based on person-specific features. This suggests that better recognition performance might be achieved by indexing each person's face based on a person-specific feature space. As a guide to further exploration of this approach, we propose the following research question: How does the performance of a face recognition algorithm based on person-specific features compare to the performance of a face recognition algorithm that indexes all faces with a common set of features?

\section{Theory}

\subsection{Gabor Filters}

Gabor wavelets are a family of filters derived from a mother Gabor function by altering the parameters of that function. The response of a particular Gabor filter is tuned to the spatial frequency, and the spatial orientation content of the region within its spatial extent. By employing Gabor filters with a variety of spatial extents, it is possible to index faces based on both large and small facial features. Because Gabor filter responses are similar to those of many primate cortical simple cells, and because they are able to index features based on their locality in both space and frequency, they have become one of the most widely chosen filters for image decomposition and representation. Gabor filters are defined as follows:

$$
\begin{gathered}
\psi_{\omega, \theta}(x, y)=\frac{1}{2 \pi \sigma_{x} \sigma_{y}} \cdot G_{\theta}(x, y) \cdot S_{\omega, \theta}(x, y) \\
G_{\theta}(x, y)=e^{-\left(\frac{(x \cos \theta+y \sin \theta)^{2}}{2 \sigma_{x}^{2}}+\frac{(-x \sin \theta+y \cos \theta)^{2}}{2 \sigma_{y}^{2}}\right)} \\
S_{\omega, \theta}(x, y)=e^{i(\omega x \cos \theta+\omega y \sin \theta)}-e^{-\frac{\omega^{2} \sigma^{2}}{2}}
\end{gathered}
$$

where, $(x, y)$ is the $2 \mathrm{D}$ spatial location where the filter is centered, $\omega$ is the spatial frequency parameter of its $2 \mathrm{D}$ sinusoidal signal, $\sigma_{d i r}^{2}$ represents the variance of the Gaussian mask along the specified direction - which can be either $x$ or $y$. This variance determines the spatial extent of the Gabor filter, where its output is readily influenced. From the definition of Gabor wavelets, as given in Equation (1), it can be seen that Gabor filters are generated by multiplying two components: (1) the Gaussian mask $G_{\theta}(x, y)$ shown in Equation (2) and (2) the complex sinusoid $S_{\omega, \theta}(x, y)$ shown in Equation (3). 


\subsubsection{The Gaussian Mask}

The 2D Gaussian mask determines the spatial extent of the Gabor filter. This spatial extent is controlled by the variance parameters (along the $x$ and $y$ directions) together with the orientation parameter $\theta$. Typically, $\sigma_{\mathrm{x}}=\sigma_{\mathrm{y}}=\sigma$. Under such conditions the orientation parameter, $\theta$, does not play any role, and the spatial extent of the Gabor filter will be circular.

\subsubsection{The Complex Sinusoid}

The 2D complex sinusoid provides the sinusoidal component of the Gabor filter. This complex sinusoid has two components (the real and the imaginary parts) which are two 2D sinusoids, phase shifted from each other by $(\pi / 2)$ radians. When combined with a Gaussian mask, the resulting Gabor filter kernel can be applied to a 2D array of pixel values (such as a region within a face image) to generate a complex coefficient value whose amplitude is proportional to the spatial frequency content of the array that lies within the extent of the Gaussian mask. If $\sigma_{x}=\sigma_{y}=\sigma$, then the real and imaginary parts of the Gabor coefficient produced by Equation (1) can be computed as follows.

$$
\left.\Re \psi_{\omega, \theta}(x, y)\right\}=\frac{1}{2 \pi \sigma^{2}} G_{\theta}(x, y) \Re\left\{S_{w, \theta}(x, y)\right\} \quad \Im\left\{_{\omega}(x, y)\right\}=\frac{1}{2 \pi \sigma^{2}} G_{\theta}(x, y) \mathfrak{I}\left\{S_{w, \theta}(x, y)\right\}
$$

\subsubsection{The Gabor feature (Coefficient)}

In order to extract a real number Gabor coefficient at a location $(x, y)$ of an image $I$, the real and imaginary parts of the filter are applied separately to the image, and the real-valued magnitude of the resulting complex number is used as the coefficient. Thus, the convolution coefficient $C_{\psi}$ at a location $(x, y)$ on an image $I$ with a Gabor filter $\psi_{w, \theta}(x, y)$ is given by

$$
C_{\psi}(x, y)=\sqrt{\left(I(x, y) * \mathfrak{R}\left\{\psi_{\omega, \theta}(x, y)\right\}\right)^{2}+\left(I ( x , y ) * \mathfrak { I } \left\{\psi_{\left.\left.\omega, \theta^{(x, y)}\right\}\right)^{2}}\right.\right.}
$$

\section{Methodology}

\subsection{Overview}

In general, feature based face recognition methods use feature detectors that are not tailored specifically for face recognition, and they make no attempt to selectively choose feature detectors based specifically on their usefulness for face recognition. The method described in this paper uses Gabor wavelets as feature detectors, but evaluates the usefulness of each particular feature detector for distinguishing between the faces within our face database. Given the very large number of possible Gabor feature detectors, we use a Genetic Algorithm (GA) to explore the space of possibilities, with a fitness function that propagates parents with a higher ability to distinguish between the faces in the database. By selecting Gabor feature detectors that are most useful for distinguishing each person from all the other people in the database, we can define a unique (i.e. person-specific) feature space for each person. 


\subsection{The Image Set}

All experiments were conducted with face images from the FacePix (30) database [12]. This database has face images of 30 people at various pose and illumination angles. For each person in the database, there are three sets of images. (1) The pose angle set contains face images of each person at pose angles from $+90^{\circ}$ to $-90^{\circ}$ (2) The no-ambient-light set contains frontal face images with a spotlight placed at angles ranging from $+90^{\circ}$ to $-90^{\circ}$ with no ambient light, and (3) The ambient-light set contains frontal face images with a spot light placed at angles placed at angels from $+90^{\circ}$ to $-90^{\circ}$ in the presence of ambient light. Thus, for each person, there are three face images available for every angle, over a range of 180 degrees.

We selected at random two images out of each set of three frontal $\left(0^{\circ}\right)$ images for training, and used the remaining image for testing. The genetic algorithms used the training images to find a set of Gabor feature detectors that were able to distinguish each person's face from all of the other people in the training set. These feature detectors were then used to recognize the test images. The same set of training and testing images were used with PCA-based face recognition, to allow a comparison with our proposed method. Figure (1) shows some example images used in our experiments.

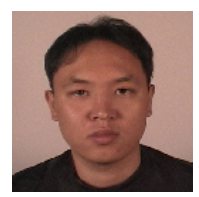

(a)

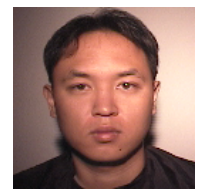

(b)

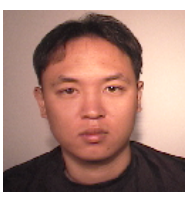

(c)

Fig. 1. (a) and (b) are the training samples of the person, while (c) is the testing sample

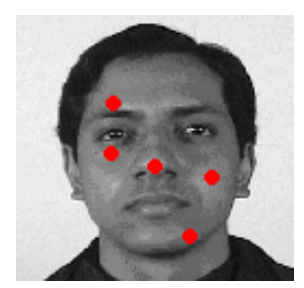

Fig. 2. A face image marked with 5 locations where unique Gabor features will be extracted 4.3 Our Gabor features

\subsection{Our Gabor Features}

Each Gabor feature corresponds to a particular Gabor wavelet (i.e. a particular spatial frequency, a particular orientation, and a particular Gaussian-defined spatial extent) applied to a particular $(x, y)$ location within a normalized face image. (Given that 125 different Gabor filters were generated, by varying $\omega, \sigma$ and $\theta$ in 5 steps each, and given that each face image contained $128 * 128=16,384$ pixels, there was a pool of 
$125 * 16384=2,048,000$ Gabor features to choose from.) We used an N-dimensional vector to represent each person's face in the database, where $N$ represents the predetermined number of Gabor features that the Genetic Algorithm selected from this pool. Fig. 2 shows an example face image, marked with 5 locations where Gabor features will be extracted (i.e. $N=5$ ). Given any normalized face image, real-valued Gabor features are extracted at these locations using Equation (5). This process can be envisioned as a projection of a 16,384-dimensional face image onto an $N$ dimensional subspace, where each dimension is represented by a single Gabor feature detector.

Thus, the objective of the proposed methodology is to extract an $N$ dimensional real-valued person-specific feature vector to characterize each person in the database. The $N(x, y)$ locations (and the spatial frequency and spatial extent parameters of the $N$ Gabor wavelets used at these locations) are chosen by a GA, with a fitness function that takes into account the ability of each Gabor feature detector to distinguish one face from all the other faces in the database.

\subsection{Our Genetic Algorithm}

Every GA is controlled in its progress through generations with a few control parameters namely, (1) the number of generations of evolution $\left(n_{g}\right),(2)$ the number of parents per generation $\left(n_{p}\right),(3)$ the number of parents cloned per generation $\left(n_{c}\right),(4)$ the number of parents generated through cross over $\left(n_{\mathrm{co}}\right)$ and (5) the number of mutations in every generation $\left(\mathrm{n}_{\mathrm{m}}\right)$. In our experiments, the GA used the following empirically-chosen GA parameters: $\mathrm{n}_{\mathrm{g}}=50, \mathrm{n}_{\mathrm{p}}=100, \mathrm{n}_{\mathrm{c}}=6, \mathrm{n}_{\mathrm{co}}=35$ and $\mathrm{n}_{\mathrm{m}}=5$.

\subsubsection{Our Fitness Function}

The fitness function of a genetic algorithm determines the nature, and the efficiency, of the search conducted within the parameter space. Our fitness function $F$ consists of an equation with two independent terms. The term $D$ is a distance measure that represents the ability of a parent (i.e. the ability of its Gabor feature detectors) to distinguish one person's face images from those of all the other people in the database. The other term $C$ represents the degree of correlation between the textural qualities of the spatial locations of the $N$ Gabor feature detectors within each parent, which are determined by applying all 125 Gabor filters to that location. These two terms are assigned weighting factors, as follows:

$$
F=w_{D} D-w_{C} C
$$

where, $w_{D}$ is the weighting factor for the Distance measure $D$, and $w_{C}$ is the weighting factor for the Correlation measure $C$.

\section{The Distance Measure $D$}

Let $M_{i}$ represent a set of Gabor features extracted for person i, where $i=1 \ldots J$ and where $J$ is the total number of people in the database. For each person $i$, let all the images of person $i$ be marked as positives, and all the other images be marked as negatives. If there are $N$ Gabor features detectors, then $M_{n, i}=\left\{m_{1, i}, m_{2, i}, \ldots, m_{N, i}\right\}$ 
represents the $N$ Gabor feature detectors, $P_{l, i}=\left\{p_{1, i}, p_{2, i}, \ldots, p_{L, i}\right\}$ represents the $L$ positive images, and $N_{k, i}=\left\{n_{1, i}, n_{2, i}, \ldots, n_{K, i}\right\}$ represents the $K$ negative images of person $i$.

The distance measure $D$ is then defined as:

$$
D=\min _{l, k}\left[\delta_{N}\left(\phi_{N}\left(p_{l, i}\right), \phi_{N}\left(n_{k, i}\right)\right)\right]
$$

Where, $\phi_{N}(X)$ is the projection of the 16,384-dimensional face image onto an $N$ dimensional subspace, where the $\mathrm{N}$ dimensions are represented by $M_{n, i}=\left\{m_{1, i}, m_{2, i}, \ldots, m_{N, i}\right\}$, and $\delta_{N}(A, B)$ is the $N$-dimensional Euclidean distance between $A$ and $B$.

\section{The Correlation Measure C}

$C$ is a penalty on the fitness of a parent that is levied if there is a correlation between the textural qualities at the $N$ spatial locations of the Gabor feature detectors of that parent. (The textural qualities of a location are determined by applying all 125 Gabor filters at that location.) This penalty is needed to suppress the GA's tendency to select multiple feature detectors within a single distinctive facial feature, such as a mustache. Application of the 125 Gabor filters to each of the $\mathrm{N}$ locations produces the following 125-column, $\mathrm{N}$ row matrix:

$$
A=\left[\begin{array}{cccc}
g_{1,1} & g_{1,2} & \ldots & g_{1, N} \\
g_{2,1} & g_{2,2} & \ldots & g_{2, N} \\
\vdots & \vdots & \vdots & \vdots \\
g_{125,1} & g_{125,2} & \cdots & g_{125, N}
\end{array}\right]
$$

Where, $g_{x, y}$ is the real-number Gabor coefficient obtained by applying the $x^{\text {th }}$ Gabor filter of the 125 -filter pool at the location of the $y^{\text {th }}$ Gabor feature detector. $C$ can now be defined as follows:

$$
C=\log (\operatorname{det}(\operatorname{diag}(B)))-\log (\operatorname{det}(B))
$$

Where, $B=\frac{1}{124} A^{T} A$ is the correlation matrix.

\section{Normalization of $D$ and $C$}

Since $D$ and $C$ are two independent measures, before they can be used in Equation (6), they need to be normalized to a common scale. For each generation, before the fitness values are computed to rank the parents, parameters $D$ and $C$ are each normalized to range between 0 and 1 . This is done as follows

$$
D_{\text {norm }}=\frac{D-D_{\text {Min }}}{D_{\text {Max }}-D_{\text {Min }}} \quad C_{\text {norm }}=\frac{C-C_{\text {Min }}}{C_{\text {Max }}-C_{\text {Min }}}
$$




\section{Results}

To evaluate the relative importance of the two terms ( $D$ and $C$ ) in the fitness function, we ran the proposed algorithm on the training set several times with 5 feature detectors per chromosome, while changing the weighting factors in the fitness function for each run, setting $w_{D}$ to $0, .25, .50, .75$, and 1.00, and computing $w_{C}=\left(1-w_{D}\right)$. Figure 3 shows the recognition rate achieved in each case.

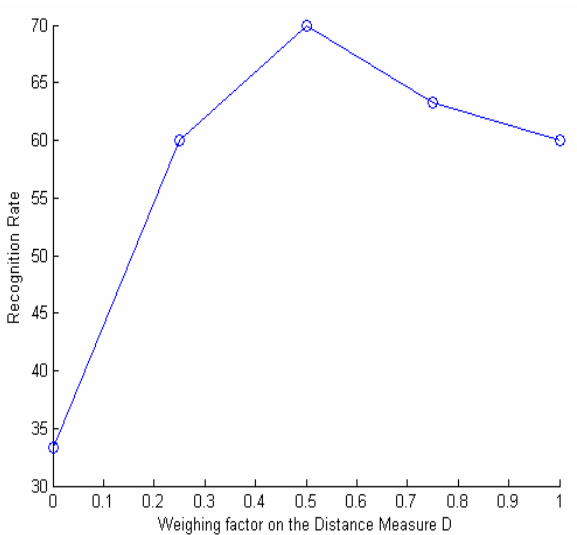

(a)

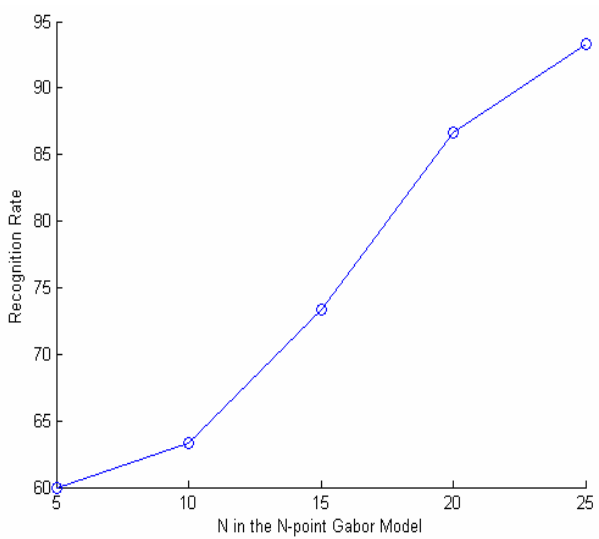

(b)

Fig. 3. (a) Recognition rate with varying weighing factor for the distance measure $D$ (b) The recognition rate versus the number Gabor feature detectors

We also ran the proposed algorithm on the training set 5 times, while changing the number of Gabor feature detectors per parent chromosome for each run to $5,10,15$, 20 , and 25 . In all the trials, $w_{D}=0.5$. Figure 4 shows the recognition rate achieved in each case.

\section{Discussion of the Results}

Fig. 3(b) shows that the recognition rate of the proposed algorithm when trained with $5,10,15,20$, and 25 Gabor feature detectors increases monotonically, as the number of Gabor feature detectors $(N)$ is increased. This can be attributed to the fact that increasing the number of Gabor features essentially increases the number of dimensions for the Gabor feature detector space, allowing for greater spacing between the positive and the negative clusters.

Fig. 3(a) shows that for $N=5$ the recognition rate was optimal when the distance measure $D$ and the correlation measure $C$ were weighted equally, in computing the fitness function $F$. The dip in the recognition rate for $w_{D}=1.0$ indicates the 
significance of using the correlation factor $C$ in the fitness function. The penalty introduced by $C$ ensures that the GA searches for Gabor features with different textural patterns. If no such penalty were to be imposed, the GA might select Gabor features that are clustered on one salient feature on an individual, such as a mole.

The best recognition results for the proposed algorithm $(93.3 \%)$ were obtained with 25 Gabor feature detectors. The best recognition performance for the PCA algorithm was reached at about 15 components, and flattened out beyond that point, providing a recognition rate for the same set of faces that was less than $83.3 \%$. This indicates that, for the face images used in this experiment (which included substantial illumination variations) the proposed method performed substantially better than the PCS algorithm.

\section{Conclusions and Future Work}

For the set of 30 face images used in these experiments (which included a wide range of illumination variations) person-specific indexing (as implemented by our proposed algorithm) provided better recognition rates than Principal Component Analysis (PCA). Furthermore (unlike PCA which flattened out after 15 components) the recognition rates for the proposed algorithm increase monotonically with increasing numbers of Gabor features. Based on Fig 4, it seems reasonable to expect that recognition rates for the proposed algorithm will continue to increase as more Gabor features detectors are added, and this will be further explored in future work. Future research will also thoroughly explore the relative importance of the $D$ and $C$ terms in the fitness function $F$ as the number of Gabor feature detectors is increased, and will evaluate the performance of the proposed method on a much larger face database.

\section{References}

[1] Holland, J. H., Adaptation in natural and artificial systems, The University of Michigan Press, 1975.

[2] Turk, M. and Pentland, A., Face Recognition Using Eigenfaces, Proceedings of IEEE Conference on Computer Vision and Pattern Recognition, 1991, pp 586-591.

[3] Etemad, $K$. and Chellappa, R., Discriminant analysis for recognition of human face images, Journal of Optical Society of America, 1997, pp 1724-1733.

[4] Lee, T. S., Image representation using 2D Gabor wavelets, IEEE Transactions on Pattern Analysis and Machine Intelligence, Vol. 18(10), Oct. 1996, pp 959 - 971.

[5] Shen, L. and Bai L., Gabor wavelets and kernel direct discriminant analysis for face recognition, Proceedings of the 17th International Conference on Pattern Recognition, 2004, ICPR 2004, Vol. 1(23-26), Aug. 2004, pp 284 - 287.

[6] Liu, C. and Wechsler, H., Independent component analysis of Gabor features for face recognition, IEEE Transactions on Neural Networks, Vol. 14(4), July 2003, pp 919 928.

[7] Liu, C. and Wechsler, H., Gabor feature based classification using the enhanced fisher linear discriminant model for face recognition, IEEE Transactions on Image Processing, Vol. 11(4), April 2002, pp 467 - 476. 
[8] Duc, B.; Fischer, S.; Bigun, J., Face authentication with sparse grid Gabor information, IEEE International Conference on Acoustics, Speech, and Signal Processing, 1997. ICASSP-97, Vol. 4(21-24), April 1997, pp 3053 - 3056.

[9] Kalocsai, P.; Neven, H.; Steffens, J., Statistical analysis of Gabor-filter representation Third IEEE International Conference on Automatic Face and Gesture Recognition, 1998. Proceedings, 14-16, April 1998, pp 360 - 365.

[10] Liu, Y. and Chongqing, Face recognition using kernel principal component analysis and genetic algorithms, Proceedings of the 12th IEEE Workshop on Neural Networks for Signal Processing, Sept. 2002, pp 337 - 343.

[11] $X u, Y$., $L i, B$., Wang, $B$., Face recognition by fast independent component analysis and genetic algorithm, The Fourth International Conference on Computer and Information Technology, 2004, CIT '04, 14-16, Sept. 2004, pp 194 - 198.

[12] Black, J., Gargesha, M., Kahol, K., Kuchi, P., Panchanathan, S., A Framework for Performance Evaluation of Face Recognition Algorithms, ITCOM, Internet Multimedia Systems II, Boston, July 2002. 